\title{
Empresarios exitosos: cómo toman decisiones
}

\author{
Castillo de Matheus, María Elizabeth
}

\section{Resumen}

En el ámbito empresarial se destacan empresarios que han garantizado un crecimiento sostenido en cuanto a oferta de productos, incremento en el número de clientes, ampliación de las zonas geográficas y en general, crecimiento empresarial. El objetivo clave de la investigación, busca interpretar hasta comprender, la estructura que subyace en el proceso de toma de decisiones de empresarios exitosos sin estudios universitarios, y de manera particular el objetivo de este artículo, es determinar las características claves de esta estructura desde el contexto empresarial, las cuales fueron obtenidas a través de un estudio cualitativo apoyado en una trama metodológica que tuvo como protagonistas a la hermenéutica, la etnometodología y las historias de vida, dando como resultado o conclusión las implicaciones que tienen la experiencia y el aprendizaje en la conformación de habilidades de tipo analítica, práctica y creativa que asociadas a los tipos de pensamiento racional, sistémico, creativo y la inteligencia emocional, conforman un decisor que con visión prospectiva, maximiza resultados en pro de organizaciones exitosas.

Palabras clave: Empresario, toma de decisiones, contexto empresarial, procesos de trabajo.

\section{Successful Entrepreneurs: How They Make Decisions}

\section{Abstract}

At the company level, businessmen who have ensured sustained growth in terms of product offerings, increase in the number of customers, expanding the geographic areas and overall business growth stand out. The key objective of this study is to interpret and understand the structure underlying the decision-making processes of successful businessmen without university studies; the special objective of this article is to determine key characteristics of this structure from the entrepreneurial context, obtained through a qualitative study based on a methodological grid whose main categories

Recibido: 20-02-10. Aceptado: 09-09-10

Docente-Investigador, Universidad Nacional Experimental del Táchira. Decanato de Investigación. Programa "Calidad y Productividad Organizacional". San Cristóbal 5001, Estado Táchira. Venezuela. e-mail: elizabeth547@yahoo.com. 
were hermeneutics, ethno-methodology and life histories. Results or conclusions were the implications that experience and learning have in shaping analytical, practical and creative skills which, associated with types of rational, systemic, creative thinking and emotional intelligence, constitute a decisionmaker who, with a prospective vision, maximizes results in favor of successful organizations.

Key words: Entrepreneur, decision making, business context, work processes.

\section{Introducción}

En el ámbito empresarial se destacan aquellos empresarios, que sin estudios universitarios, han demostrado en el manejo de sus organizaciones de manera distintiva la competencia emprendedora, la realidad confirma que en el sector empresarial tachirense, existen empresarios emprendedores y exitosos sin formación universitaria. Esta situación se evidencia en el Informe de Avance del Programa Calidad y Productividad Organizacional (Castillo, 2002), que expone que el $60 \%$ de los empresarios encuestados, no poseen estudios universitarios. Sin embargo, estos empresarios han sabido tomar decisiones en el momento adecuado, sobre el tópico conveniente, y con la firmeza necesaria para poder generar un futuro exitoso.

En función a este planteamiento se generó una investigación orientada a profundizar el conocimiento sobre empresarios con estas características, centrando el objetivo del estudio en el proceso de decisiones. Énfasis que se asume considerando las opiniones de expertos en el área administrativa, quienes comparten la opinión de que éste proceso es la clave de la planeación, premisa fundamental del éxito empresarial. Este proyecto se denominó: Una Teoría sobre el Proceso Decisorio de Empresarios Exitosos sin Estudios Universitarios, el cual analizó como punto focal las decisiones tomadas por los entrevistados en tres diferentes ámbitos: el contexto empresarial, el contexto familiar y el contexto individual.

Este artículo presenta una parte sustancial de los resultados obtenidos en el proyecto de investigación citado, enfocado en el contexto empresarial en el cual surgieron, luego del análisis de los relatos, cuatro categorías emergentes: los procesos de trabajo, la capacidad de riesgo, la posición ante la pérdida y la concepción del trabajo; cada uno de los cuales se abordó en detalle para descifrar los aportes individuales y conjuntos al proceso decisorio llevado a cabo por los informantes. Este artículo se enfoca en los resultados obtenidos en el contexto empresarial, específicamente en la categoría procesos de trabajo.

A nivel general el objetivo clave de la investigación, busca interpretar hasta comprender, la estructura que subyace en el proceso de toma de decisiones de empresarios exitosos sin estudios universitarios, y de manera particular el objetivo de este artículo, es determinar las características claves de esta estructura desde el contexto empresarial. Este planteamiento implica la utilización del paradigma del descubrimiento cualitativo-interpretativo, y dentro del mismo el enfoque hermenéutico que proporciona un proceso de comprensión en una espiral que va del todo a las partes y de las partes al 
todo, adquiriendo sentido y significado. La hermenéutica proporciona herramientas para la interpretación de los relatos, los cuales se analizan de manera particular en cuanto a las narraciones individuales y luego se conjugan con las emitidas de manera general interpretándolas en un marco de referencia contextual, conectándolas posteriormente con el sentido que posee en el marco general de su actuación como empresario exitoso. Se busca interpretar la acción del decisor, indagar sobre su intención, estableciendo una relación empática con el entrevistado a fin de descubrir las pautas que guían su desempeño.

El proceso decisorio es un proceso interno, observable en las consecuencias expresadas en acciones; es por esta razón que resulta evidente su estudio con un método que propenda a la búsqueda de las razones de sentido de las acciones personales. La etnometodología proporciona las herramientas fundamentales para que se evidencie esta visión del proceso. La etnometodología se centra en la interacción verbal, analizando el discurso a través de la semiótica, privilegiando al diálogo como instrumento para la recogida de datos, los cuales se registran a través de sistemas de audio, video y notas de campo. Su raíz disciplinaria se encuentra en la sociología y la corriente de pensamiento que le dio origen se fundamenta en la fenomenología y el interaccionismo simbólico (Sandín, 2003).

Un método que se considera, operacionaliza los dos anteriores en la tarea propuesta, es el de historias de vida, método utilizado en investigación como una manera eficaz, segura y confiable para la recogida de datos y escudriñar la vida cotidiana de los informantes con sus características y particularidades.

Las unidades de información, se conformaron con empresarios exitosos que no poseían estudios universitarios y cuyas organizaciones están localizadas en el estado Táchira. La característica fundamental de esta población, está en función del éxito empresarial, definido para este caso particular, como el desempeño exitoso que puede ser evidenciado en el crecimiento organizacional ya sea en infraestructura física, en número de productos ofertados o líneas de producción, ampliación de las zonas atendidas o cualquier otro indicador cuantitativo mensurable en los últimos 5 años, período que, según el Observatorio Pyme (2001), es el requerido para la consolidación empresarial. Los empresarios fueron seleccionados como muestra intencional, preservandose su identidad, y entrevistados en el período de enero del año 2001 a junio del 2004, espacio de tiempo en que se desenvolvió el proceso investigativo.

Se eligió como técnica de recolección de información, las entrevistas en profundidad. Técnica que permite al entrevistador obtener información sobre determinado problema y, a partir del mismo, establece una lista de temas en relación con los que se focaliza la conversación, la cual debe ser profunda y no directiva, quedando ésta a libre elección del entrevistador, quien podrá sondear razones y motivos o ayudar a establecer determinados factores, pero sin ajustarse a una estructura formalizada de antemano. 


\section{Toma de decisiones en el contexto empresarial}

Del análisis minucioso de los relatos de los entrevistados, surgen inicialmente los contextos de acción, dentro de los mismos se generan categorías, que en el caso particular del contexto empresarial, analizado en este artículo, hace referencia a los resultados obtenidos en la categoría: procesos de trabajo. Dentro de las categorías, y en función del análisis de los relatos, se destacan algunas concepciones, opiniones, comentarios o acciones, que comparten cualidades específicas las cuales se definen como conceptos básicos, estos primeros constructos se someten a un procesamiento y se agrupan de acuerdo a su naturaleza, generando concepciones de mayor significación teórica denominados conceptos emergentes. En el caso de la categoría Procesos de Trabajo, este análisis arrojó los resultados presentes en el Cuadro 1.

\section{Conceptos Emergentes}

Los conceptos básicos numerados del 1 al $5(1,2,3,4$, y 5$)$, tienen que ver con los conceptos referentes a las áreas contable y económica, la primera de las cuales es denominada por autores como Horngren (1989:16), como el "lenguaje de las decisiones financieras" que unida a la definición de economía presentada por Martínez (2001:103), en la cual circunscribe su campo de acción a todos los problemas que tienen que ver con la determinación de precios y costos que producen beneficios medibles en dinero en la producción de bienes y servicios, conjugan el manejo de información referente a ambas áreas materializada en conceptos como los de costos, gastos, precios, utilidad e inversiones, los cuales influyen de manera determinante en la toma de decisiones.

Del análisis de los relatos relacionados al área contable-económica, surgen como conceptos básicos: la conciencia clara de la estructura de costos; el manejo de la relación costo, precio, utilidad; la iniciativa en el establecimiento y control de costos, la optimización de la inversión como objetivo económico-financiero y la visión de la inversión como forma de crecimiento. El manejo de estos conceptos y concepciones son fundamentales para

\section{Cuadro 1}

Contexto Empresarial. Categoría Procesos de Trabajo

Contexto empresarial

Conceptos Básicos

Comentarios Generales

Procesos de trabajo

\begin{tabular}{|c|c|c|}
\hline Conceptos Básicos & Comentarios Generales & $\begin{array}{l}\text { Conceptos } \\
\text { Emergentes }\end{array}$ \\
\hline $\begin{array}{l}\text { 1. Estructura de costos. } \\
\text { 2. Relación costo-precio-utilidad } \\
\text { 3. Iniciativa en el control de } \\
\text { precios, costo y utilidad. } \\
\text { 4. Optimización en la inversión } \\
\text { 5. Inversión para el crecimiento. }\end{array}$ & $\begin{array}{l}\text { Los entrevistados poseen una clara conciencia de } \\
\text { estructura de costos de la empresa para cada tipo de } \\
\text { producto y su incidencia en los precios y utilidad. Esta } \\
\text { conciencia los orienta a toma de decisiones claras } \\
\text { sobre las inversiones, las cuales apuntan hacia el } \\
\text { crecimiento organizacional, teniendo en mente la op- } \\
\text { timización de los recursos económicos. }\end{array}$ & $\begin{array}{c}\text { Información } \\
\text { Económica- } \\
\text { Financiera. } \\
\text { Visión } \\
\text { Prospectiva. } \\
\text { Optimización. }\end{array}$ \\
\hline
\end{tabular}




\section{Cuadro 1 (Continuación)}

Contexto empresarial
Procesos de trabajo
Comentarios Generales

Conceptos

Emergentes

6. Importancia del empleado

7. Conocimiento de cargos y funciones

8. La familia empleados

9. Alianza con empleados

10. Iniciativa en atención a clientes

11. Necesidades del cliente

12. Atención al cliente.

13. El conocimiento del Producto y su Calidad, clave del negocio.

14. Monitor de distintas áreas.

15. El lugar de trabajo es la empresa, no la oficina.

16. Aprendizaje permanente.

17. Aprendizaje clave para las decisiones.

18. Experiencia clave del aprendizaje.

19. Conexiones e interconexiones del sistema.

20. Planteamientos creativos
Hay consenso en el papel preponderante que juegan los empleados en el desarrollo del negocio, por eso los entrevistados a lo largo de los relatos destacan esta importancia y las distintas maneras en que cada una de sus organizaciones los atiende y organiza. Es de destacar que la permanencia del empleado en la organización está supeditada al grado de empatía que logre con el dueño. La familia es vista como empleados dentro del contexto empresarial.

Consideración del cliente como elemento fundamental para el negocio. Los entrevistados coinciden en que son la clave del desarrollo empresarial, por eso se conoce, se atienden sus necesidades y se asesora buscando su comodidad y satisfacción.

Los entrevistados están claros que la calidad de su producto es una de las claves fundamentales del negocio, por eso asumen acciones concretas respecto al mejoramiento de las cualidades del producto, incluyendo dentro de las mismas la oportunidad de entrega y el precio.

El conocimiento al detalle del proceso y su monitoreo permanente distingue a los entrevistados quienes no se consideran gerentes de oficina y se comportan como gerentes de proceso.

La información obtenida de las distintas áreas de la organización es procesada de manera permanente convirtiéndose en un aprendizaje constante, donde la experiencia juega un papel fundamental.

El manejo de información de las distintas áreas de la empresa, de los clientes y los mercados se conjuga en un todo sistémico que hace a los entrevistados asumir este pensamiento para tomar decisiones

La originalidad juega un papel importante para los entrevistados que usan indistintamente en muy diversas acciones para alcanzar distintos objetivos.
Información referente a los empleados Empatía.

Trabajo en equipo. La familia como empleados.

Información referida al cliente.

Atención y satisfacción del cliente.

Calidad. Información sobre el producto.

Información del proceso. Iniciativa.

Monitoreo del proceso.

Aprendizaje. Experiencia.

Pensamiento sistémico.

Creatividad.

Fuente: Propia (2005). 
los entrevistados al momento de tomar decisiones. Su agrupación y consolidación dio como resultado una categoría de mayor significación denominada conceptos emergentes constituidos por la información económico-financiera, la visión prospectiva y la optimización.

El manejo de datos económico financieros se realiza con una visión de anticipación, con una reflexión que ilumina la acción en pro del futuro. Esta forma particular de conducción por parte de los entrevistados, destaca su visión prospectiva definida por autores como Mojica (1991) y Gabiña (1996), como la identificación de un futuro probable, deseable y posible que depende del conocimiento $y$ manejo de la información pertinente, que los prepara para afrontar con flexibilidad y anticipación los retos del futuro. Como lo plantea Godet (1995:3), "es una reflexión para iluminar la acción presente con la luz de los futuros posibles". Existe sincronización entre información económico-financiera, visión prospectiva y optimización que apunta hacia decisiones efectivas.

Los conceptos básicos tomados del Cuadro 1, identificados por los números 6, 7, 8 y 9 (importancia del empleado, conocimiento de cargos y funciones, la familia como empleados y alianza con empleados), dan cuenta del consenso que existe entre los informantes referente a la importancia de los empleados y su papel fundamental en el desarrollo del negocio. Los comentarios destacan la manera en que cada organización los atiende y organiza, y acentúa la importancia de que los empleados logren una relación estrecha con el dueño a fin de garantizar su permanencia en el contexto em- presarial. Es importante destacar que la familia forma parte de este contexto y que la misma se asume como parte de la nómina de empleados. Estos conceptos básicos dan origen a nuevos conceptos emergentes que en esta ocasión se asocian a la Información referente a los empleados, empatía, trabajo en equipo y la familia como empleados.

La información, constituida por "datos que se han organizado o analizado de acuerdo con alguna lógica" (Stoner et al., 1998:G-6), está en esta oportunidad referida al conocimiento de los empleados en sus cargos y funciones que amerita una clara comprensión de las tareas llevadas a cabo por cada uno de ellos y su aporte a la eficiencia de la organización. Este enfoque lleva a los informantes a involucrarse de manera muy especial con los empleados, propiciando la aparición del concepto de empatía, que según Goleman (1997:250), es una capacidad o habilidad especial que se traduce en el conocimiento de las emociones del otro, sus deseos y aspiraciones que da la posibilidad del estrechamiento de las relaciones interpersonales, o de manera concreta: es "la capacidad intuitiva para comprender las emociones".La permanencia del empleado en la organización está supeditada al logro de un grado de empatía con el dueño, quien a través de esta vía establece alianzas y coaliciones con los empleados que comparten ambiciones, deseos y esperanzas de corte similar a las propias. Esta sintonía emocional, es imprescindible según Märtin (1998), para la conformación de equipos. El trabajo en equipo, según esta autora, constituye el pilar fundamental para el éxito empresarial, es indispensable que los empleados estén 
dispuestos a integrarse con sus colegas para tirar junto a en la misma dirección y esto se logra si existe un alto grado de cohesión.

Las interrelaciones que se dan entre los conceptos emergentes de esta área, propician que surja la consideración de la familia como empleados, dado el nivel afectivo que poseen con el propietario. Sus condiciones de trabajo se asemejan a la de los demás empleados aunque en ocasiones poseen ciertos privilegios que les permite asumir para sí ciertas decisiones, aunque, al igual que ocurre con los demás trabajadores, el grado de libertad que le es concedido está supeditado al grado de empatía que poseen con el empresario.

Siguiendo la información presente en el Cuadro 1, los conceptos básicos: iniciativa en la atención, necesidades y atención al cliente, señalados con los números 10,11 y 12 ; destacan el lugar de privilegio que proporcionan los informantes a las personas que adquieren sus productos, enfocándose en el conocimiento de sus particularidades, necesidades y gustos a fin de establecer formas concretas de atención y satisfacción. Surgen en este caso dos conceptos emergentes relacionados con los entes privilegiados por los entrevistados: Información referida al cliente y atención y satisfacción del cliente.

Los empresarios exitosos poseen un interés particular en atender y satisfacer a su cliente, definido por Hernández (2002:75), como la persona o ente que compra o utiliza los productos/servicios de una organización en función de prerrequisitos preestablecidos y que constituye el eje fundamental de su desarrollo. La información referida al cliente garantiza su pronta ubicación, cuantificación, clasificación y la determinación de sus deseos y necesidades, requisito indispensable para poderle brindar atención y satisfacción, la cual, según Salgado (2000:21), es "la percepción del cliente sobre el grado en que se han cumplido con sus requisitos" es un concepto íntimamente ligado al significado de calidad propuesto por Harrington (1990:41), que define como "el cumplimiento o superación de las expectativas del cliente a un costo que le represente valor". Según estas definiciones, atender al cliente no es solamente proporcionarle un trato cálido y efusivo, sino que requiere un conocimiento del mismo con un grado de profundidad que dé constancia de la comprensión de cuáles son sus expectativas respecto a las características del producto.

Otra área del proceso de trabajo que surge de los relatos, es la referida al producto, evidenciada en el concepto básico: conocimiento del producto y su calidad clave del negocio, identificado con el número 13, respecto del cual los informantes no solo poseen un amplio conocimiento, sino que además se preocupan por sus atributos representados por sus características físicas, la oportunidad de entrega y su precio. Los conceptos emergentes en esta ocasión están representados por la Información del producto y calidad.

El producto definido por Stanton (1991) y Kotler (1992), como insumos transformados en función de las necesidades del cliente cuya presentación puede ser tangible o intangible, representa la razón de ser de las organizaciones sociales que se encargan de su fabricación, distribución o prestación (en el caso de servicios), debe ser desde este razo- 
namiento, punto focal de análisis por parte de los informantes, quienes poseen una clara conciencia de sus características, cantidades, oportunidad de venta, precio y cualquier otra particularidad perceptible que induzca al cliente a su compra. Esta definición de producto y la clara conciencia de lo que representa para la organización, está íntimamente ligada al concepto de calidad manejado desde el punto de vista estratégico, el cual hace énfasis en las necesidades del consumidor e incorpora el precio, la oportunidad para la compra y el servicio post venta como componentes esenciales de la misma, según lo expresado por Escorche et al. (1995:41). La calidad en definitiva, es una cualidad del producto percibida por el cliente y que puede en función a sus expectativas inducir o no a su compra, convirtiéndose desde esta perspectiva, en el punto de enlace entre cliente y producto.

Los empresarios exitosos, conscientes de la importancia del producto se interesan en conocer el proceso para su elaboración, esta capacidad les da la posibilidad de intervenir en cualquiera de sus partes y convertirse en seguidores y controladores del proceso según lo expresado en los conceptos básicos: monitor de las distintas áreas, y el lugar de trabajo es la empresa no la oficina, señalados en los numerales No 14 y 15 . Estos comentarios sustentan los conceptos emergentes, Información del proceso, iniciativa y monitoreo del proceso.

El proceso definido como la secuencia sistemática de actividades para la elaboración de un producto o servicio, o como lo conceptualiza Niebel (1996), secuencia de acciones encadenadas que trasforman en productos o resultados con características definidas, unos insumos o recursos variables agregándoles valor con un sentido específico, representa otra de las claves de importancia fundamental en una organización social que impacta su sistema de decisiones.

La información sobre el proceso, debe contener una clara identificación de las actividades, su secuencia e interrelaciones y el impacto que cada una posee en las operaciones de transformación. Los empresarios exitosos manejan a la perfección el proceso referente a su organización en todas y cada una de sus partes, como consecuencia de la familiarización que han tenido con el mismo al haber participado en el diseño de las actividades que lo conforman.

Consecuentemente, el manejo de información propicia acciones concretas y oportunas para resolver situaciones que se presentan en el desarrollo del proceso, las cuales son asumidas de manera natural por los informantes y representa una cualidad denominada iniciativa, o como la denomina Covey (1993), el hábito de la proactividad, que garantiza la victoria personal al hacer al individuo dueño de la manera cómo reacciona a los estímulos ambientales.

El manejo de información sobre el proceso y su desarrollo, desencadenan funciones de control traducidas en el monitoreo del proceso, que en palabras de Gosling (1995:91), tiene como objetivo evaluar la manera en que se desenvuelve y se alcanzan los resultados y que están en relación directa con la efectividad con la que se implementa una actividad. EI control permite garantizar que las actividades reales se ajusten a las planificadas 
permitiendo accionar rápidamente si se detecta alguna desviación.

Los conceptos emergentes que han surgido hasta el momento dentro del contexto empresarial asociados a los procesos de trabajo, evidencian el amplio sistema de información que poseen los entrevistados de todas las áreas que lo conforman, esta información no es solo un insumo que se ingiere para tomar decisiones, sino que la misma es procesada generando un aprendizaje que es en definitiva el que direcciona cualquier decisión. Los conceptos básicos: aprendizaje permanente, aprendizaje clave para las decisiones y experiencia clave para el aprendizaje (No 16, 17 y 18), refuerzan esta afirmación agregando un ingrediente al proceso: la experiencia. Este razonamiento converge en la aparición de los conceptos emergentes aprendizaje y experiencia.

El aprendizaje definido por Robbins (1999:68), como "cualquier cambio relativamente permanente en el comportamiento que ocurre como resultado de la experiencia" o como lo conceptualiza Anderson (2001:5), "es el proceso por el cual ocurren cambios duraderos en el potencial conductual como resultado de la experiencia" sugiere que no es posible ver a alguien aprendiendo, sin embargo, observando los comportamientos de las personas se puede determinar si en ella ha ocurrido un proceso de aprendizaje.

Dadas las características de la investigación, las de sus informantes, y la posición propia de la autora respecto a los procesos de aprendizaje, se asume la teoría del aprendizaje social como fundamento conceptual para el análisis de estos constructos procedentes de los rela- tos. Esta teoría propuesta por Bandura (1983), constituye un esfuerzo por presentar un marco de referencia integrador para analizar la conducta humana. Esta concepción no considera al individuo dominado por factores internos, ni determinado y controlado por estímulos externos, sino que se explica como un modelo de reciprocidad triádica en el que la conducta, los factores personales direccionados por los procesos cognitivos, y los acontecimientos ambientales actúan entre sí de manera interactiva. Bajo esta concepción las personas pueden considerarse como agentes causales de su propia conducta, dado que las mismas afectan el ambiente mediante sus acciones y este, a la vez, afecta su conducta de manera recíproca, accionar que es sustentado por el principio de recursión propio del pensamiento complejo.

Las personas no se limitan simplemente a reaccionar frente a un entorno inmediato, la mayor parte de las conductas está regulada por las previsiones. La perspectiva del futuro confiere a las personas la capacidad de predecir las consecuencias más probables de sus acciones, se ponen metas a sí mismas y planifican cursos de acción para eventos futuros previstos. Esta posibilidad de anticipación actúa como ente motivador al hacer conexión con las expectativas que el individuo posea a cerca de los resultados, tal como lo plantea Contreras (2001), quien además afirma que a la hora de realizarse una conducta, la persona considerará los incentivos o beneficios que resultarán de la misma.

Los datos obtenidos en la investigación, patentizan el aprendizaje como concepto emergente evidenciado en la 
conducta de los informantes. Estas conductas explicadas con la teoría social cognitiva, justifican el planteamiento fundamental de la teoría que hace referencia al aprendizaje por observación y por experiencia directa. Los relatos muestran claramente como los informantes se nutren de terceros y de sus propias prácticas para mejorar la manera de conducción de su negocio, tareas que realizan de manera permanente y con objetivos claramente definidos.

El aprendizaje para el caso estudiado, es producto de la observación y de la experiencia directa, mediado por procesos cognitivos que determinan los eventos que son factibles de ser observados, como serán percibidos y la información clave en función del valor que la misma posee para el objetivo que previamente se ha determinado. Los informantes coinciden en la obtención y observación detallada de información referida al área económica financiera, a los empleados, al cliente, al producto y al proceso en una clara orientación hacia las aristas que conforman la base fundamental de un negocio. El procesamiento de esta información, ha generado algunas formas de laborar innovadoras, como la propensión al trabajo en equipo, la empatía, la atención y satisfacción al cliente, que a la vez, se convierten en insumos fundamentales en el proceso de aprendizaje. El mismo Bandura (1983:68), lo confirma cuando dice que "los factores que conforman la tríada, pueden ser al mismo tiempo estímulo, respuesta o reforzador, dependiendo el momento arbitrario para iniciar el análisis".

El aprendizaje posee una íntima relación con la experiencia, dado que ésta es la principal fuente de instrucción para los informantes. La relación aprendizajeexperiencia está mediada por procesos cognitivos, entre ellos la memoria, tal como lo expresa Anderson (2001:6), es "el registro de la experiencia que subyace en el aprendizaje". El término memoria, depende de la definición de aprendizaje y agrega a ésta el término de registro, considerado como el cambio mental que debe materializarse para que la experiencia se convierta en un verdadero aprendizaje. El registro de memoria, es bajo esta perspectiva, el responsable de que la experiencia se convierta en aprendizaje.

Siguiendo con el análisis de los relatos, aparece un tipo especial de procesamiento de la información asociada a una habilidad que poseen los entrevistados de realizar conexiones e interconexiones entre las diferentes partes del sistema, mostrado en el concepto básico $\mathrm{N}^{0}$ 19. Esta capacidad de razonamiento surge como concepto emergente denominado Pensamiento sistémico.

Este concepto emergente, presenta un mayor grado de elaboración que los anteriores referente a procesos complejos asociados al pensamiento. El pensamiento, definido en función de Berlyne (1982), Sánchez (1994) y Albornoz (1997), que coinciden en enunciarlo como cualquier proceso del entendimiento y la razón que genera respuestas simbólicas que cumplen una función y que conducen a la solución de problemas. Está asociado a procesos que facilitan el almacenamiento, recuperación y uso adecuado del conocimiento en función de mejorar procesos de aprendizaje que se manifiestan en la obtención de habilidades para la planificación, búsqueda y 
comprensión de la información, resolución de problemas y para la toma de decisiones. Por otra parte, sistema definido por Daft (2000) y Hernández (2002), como un conjunto de elementos, conformados como un todo, que interactúan entre sí con un fin determinado. Tomando estas definiciones, el pensamiento sistémico puede conceptualizarse como el proceso del entendimiento y la razón que genera respuestas simbólicas para la resolución de problemas, tomando en consideración los distintos elementos que lo constituyen y las interrelaciones que entre los mismos se generan, dando como resultado decisiones adecuadas al todo dentro de un contexto particular.

El pensamiento sistémico, es una capacidad especial que poseen los empresarios exitosos seleccionados, quienes a lo largo de los relatos destacan la manera en que enfrentan distintas situaciones y generan soluciones, utilizando informaciones de muy diversas fuentes, para demostrar de esta manera, según lo expresado por el Instituto Andino de Sistemas (2002), una percepción especial del mundo real en términos de totalidades para su análisis y comprensión.

De igual manera los empresarios exitosos se valen de toda una suerte de estrategias para lograr algunos objetivos en situaciones particulares poniendo en evidencia una manera original de aprovechar oportunidades, el concepto básico $\mathrm{N}^{0} 20$ relacionado con la creatividad, expresa con claridad esta destreza, dando origen al concepto emergente denominado Creatividad.

La creatividad es un concepto ligado a diversas representaciones que van desde las asociadas a procesos de des- cubrimiento, a revelaciones e inspiración, así como también a concepciones más elaboradas relacionadas con el desarrollo científico y tecnológico. Dentro de esta última categoría se encuentran las definiciones proporcionadas por Guilfod (Citado por Sánchez, 1999:221), que enfatiza que la creatividad está asociada a la fluidez o capacidad para generar ideas, la habilidad para seleccionar soluciones a problemas, la originalidad relacionada con la generación de soluciones únicas y nuevas a los problemas. Por otra parte, el concepto aportado por Torrence (Citado por Sánchez, 1999:12), la define como "el proceso de percibir problemas o lagunas de información, formular ideas o hipótesis, verificar estas hipótesis, modificarlas y comunicar sus resultados". Estos autores coinciden en que la creatividad es uno de los factores que más contribuye a la solución de problemas, objetivo de las decisiones.

Esta capacidad especial de percepción se manifiesta en la solución que los informantes les dan a las distintas situaciones problemáticas que a menudo se le presentan en su actividad laboral, y es el fundamento primordial para que se manifiesta la habilidad y el pensamiento creativo.

\section{Aproximación a un modelo de toma de decisiones}

Al analizar los conceptos emergentes que son producto de los procesos de trabajo; información, trabajo en equipo, empatía, atención y satisfacción al cliente, la familia como empleados, creatividad, aprendizaje y experiencia, y pensamiento sistémico, puede notarse que los 
mismos no poseen el mismo grado de elaboración conceptual, en este caso por ejemplo, se pueden situar los procesos de información y el pensamiento sistémi$\mathrm{co}$, donde obviamente los niveles de abstracción requeridos para cada uno varían en grado de complejidad. Asociados a estos niveles de complejidad, se generan procesos intermedios que conectan fácilmente y justifican la aparición de los niveles de complejidad mayor. Consecuencia de este razonamiento, los conceptos se agrupan de acuerdo al criterio complejo de menor a mayor grado, interrelacionándose con otros conceptos que ayudan a comprender los componentes del sistema de decisiones.

Se realizó una agrupación de los conceptos emergentes de acuerdo con tres niveles formulados como: conceptos emergentes de 1er orden; constituidos por las concepciones generales de menor grado de elaboración relacionados directamente con los datos, que al ser agrupadas en conceptos de superior especificidad producto de su procesamiento, generan concepciones de mayor grado de integración denominados conceptos emergentes de 2do orden, los cuales a su vez son ubicados dentro de estructuras de significación de carácter global que buscan un mayor nivel de consolidación, generando conceptos emergentes de 3er orden, son los que poseen el grado de abstracción de mayor nivel.

\subsection{Conceptos emergentes de 1er orden}

Los conceptos emergentes de 1er orden están constituidos por concepciones bien diferenciadas y que aportan sig- nificativamente al estudio del proceso decisorio como lo son las nociones de información, de trabajo en equipo, empatía, atención y satisfacción del cliente, la familia como empleados y creatividad.

La información, se retoma nuevamente desde una perspectiva globalizadora que la coloca en el contexto preciso de la toma de decisiones. Este concepto, según lo expresado por Stoner et al. (1996:103), no es solo los datos constituidos por cifras y hechos crudos de determinado aspecto de la realidad, sino el resultado de haber organizado y analizado los mismos de alguna manera y con algún propósito que permite a los empresarios exitosos poseer un amplio panorama sobre lo que ocurre en la organización y fuera de ella, proporcionándole una visión clara de las acciones a emprender en función de alcanzar las metas trazadas. Otros conceptos aparecen dentro de este mismo nivel de abstracción conformados por el trabajo en equipo, la empatía, atención y satisfacción al cliente, la consideración de la familia como empleados y la creatividad, los cuales conforman un todo organizado que afecta los procesos de aprendizaje de los empresarios exitosos.

Todos los conceptos emergentes de primer orden son tratados por los procesos cognitivos de los empresarios exitosos, quienes por medio de la capacidad de simbolización pueden seleccionar los significados que desean dar a estos insumos. Estos, son afectados por las capacidades de previsión y autorregulación que actúan como filtros que determinan las entradas que serán seleccionadas, como serán percibidas y cuál es el impacto que tendrán en las acciones concretas que se producen como consecuencia de las de- 
cisiones asumidas por el empresario. Media también en este proceso la capacidad de autorreflexión que conjuntamente con la experiencia, orienta las acciones en pro de la optimización de los recursos buscando el crecimiento organizacional, destacándose así la visión prospectiva de los empresarios exitosos.

Este razonamiento destaca como los factores considerados, influencian y son influenciados para generar un determinado comportamiento traducido en proceso de aprendizaje y experiencia, los cuales han sido producto de la adecuación de las estructuras internas del individuo para captar y analizar una situación particular. Esta reflexión implica que los empresarios exitosos considerados como sujetos que aprenden, realizan conscientemente un procesamiento de información que les permite la adquisición de estructuras de conocimiento que han sido mediados por sus procesos cognitivos y por estímulos externos. De acuerdo con estos planteamientos, Benjafield (1997:23), hace referencia a que el aprendizaje se da dentro de un complejo sistema que incluye el percibir, recordar, procesar, crear y resolver problemas.

Se asumen como conceptos de primer orden los relacionados con los procesos de información, y los incentivos multiformes: trabajo en equipo, empatía, atención y satisfacción al cliente, la consideración de la familia como empleados y creatividad, los cuales generan y a la vez son generados por los procesos de aprendizaje y experiencia, que se traduce en una relación compleja donde los distintos componentes se entrelazan conformando una trama donde todos los elementos son generados y generadores de las distintas partes del sistema.

El aprendizaje que tiene lugar en los empresarios exitosos está caracterizado por procesos cognitivos que evidencian la aparición de habilidades de tipo analítica, práctica y creativa, lo cual se justifica si se asume que las habilidades alcanzadas por un individuo son producto de su proceso de aprendizaje, tal cual lo afirma Sánchez (1998:17), quien sostiene que es éste el responsable de las capacidades que desarrolla el individuo para procesar información, recuperarla y adecuarla a la solución de problemas y toma de decisiones.

\subsection{Conceptos emergentes de 2 do orden}

El aprendizaje se patentiza en habilidades clasificadas en analíticas, prácticas y creativas; las cuales están asociadas a los distintos procesos que conforman los conceptos emergentes de primer orden, así la información se asocia a las habilidades tanto analítica como práctica, los otros conceptos emergentes están asociados a la habilidad práctica, con excepción de la creatividad que se relaciona con la habilidad del mismo nombre. Relacionando los conceptos emergentes de primero y segundo orden con el proceso decisorio, se establece una interacción donde las habilidades analítica, práctica y creativa se conectan a la toma de decisiones pero afectadas y a la vez afectando el aprendizaje y la experiencia, enlazadas imbricadamente con los conceptos emergentes de primer orden. Este todo interactúa de manera permanente, nutrién- 
dose unos a otros en una espiral que confluye en una decisión.

La habilidad conceptualizada por Anderson (2001:448), como "conocimiento procedimental de la forma de ejecutar una tarea", o como la define Robbins (1999:746), "capacidad para enfrentar distintos tipos de situaciones", hacen mención de una capacidad especial para realizar una actividad, y es según Anderson (2001:327) "el proceso de adquisición de fluidez en el uso del conocimiento", destaca además un procesamiento y acción, en ocasiones, automático e inconsciente, este mismo autor afirma que una habilidad se desarrolla pasando por la etapa cognitiva, la etapa asociativa y la etapa autónoma. Las habilidades analíticas según Sternberg (1994), son necesarias para comparar, contrastar y evaluar las opciones que se tienen en la vida, proporcionan la capacidad para identificar un problema, definirlo, diseñar una estrategia para resolverlo y monitorear el proceso de resolución. Las habilidades prácticas se evidencian en el mundo real en el día a día, a través de la implementación de opciones funcionales. Un aspecto fundamental de este tipo de habilidad es la capacidad para entender y usar el conocimiento tácito, el cual hace referencia al conocimiento orientado a la acción que es adquirido de manera directa, sin la ayuda de otros y que permite a los individuos lograr objetivos que valoran personalmente. La habilidad creativa interviene en la creación, la invención, el descubrimiento, la imaginación y en el ir más allá de la información suministrada. Una persona creativa genera ideas novedosas de alta calidad, acorde a la tarea que desempeña y a las situaciones a las que se ve expuesto.

Estas habilidades, la analítica, práctica y creativa, conforman según Sternberg (1998), las capacidades propias de lo que él ha denominado inteligencia de éxito o exitosa, la cual define como la capacidad intencional para adaptarse a diferentes ambientes, moldearlos y seleccionarlos para lograr propósitos personales o los de una sociedad y cultura; esta forma de percibir la inteligencia está dentro de las definiciones que existen sobre el particular y que coinciden en representarla a través de habilidades, según Radler (2001), la inteligencia está compuesta de muchas habilidades o capacidades mentales distintas que operan de manera más o menos independiente.

Sternberg (1997:26), realiza una clara caracterización de los atributos que presentan las personas con esta inteligencia, resaltando que el éxito no está supeditado a poseer un elevado coeficiente intelectual y que el mismo solo garantiza buen desempeño escolar; afirma que "el cociente intelectual es un elemento pobrísimo de predicción de los logros en la vida", asegurando que solo evalúa la capacidad analítica y no toma en consideración otras habilidades propias de la inteligencia. El autor distingue a las personas con inteligencia exitosas como: (a) proactivas en la detección, definición y solución de problemas; (b) poseen visión de largo plazo; formulan la estrategia de resolución de problemas tomando en consideración objetivos de largo alcance;

(c) Poseen capacidad especial para el manejo de la información y le dan significado en función a sus expectativas; 
(d) tienen clara concepción de lo que son recursos, su manejo, uso y costo, para lo cual manipulan información de tipo económico financiera, lo que les confiere una cualidad especial para medir el riesgo de las acciones en las cuales siempre busca la maximización de resultados; (e) están conscientes de que ellos y otros pueden cometer errores y los asumen como parte del proceso de aprendizaje; $(f)$ poseen un alto grado de racionalidad matizada con intuición y (g) ostentan una clara concepción del producto y su proceso de elaboración con clara orientación hacia la obtención de resultados. Los pensamientos los traducen en acciones en función de resultados claramente definidos $y$, a pesar de ser independientes, poseen una clara orientación hacia el trabajo en equipo rodeándose de personas que comparten sus intereses y con las cuales logra una conexión afectiva.

Las características de las personas exitosas, evidencian que son ingeniosas para evaluar problemas y generar soluciones inesperadas, analizar situaciones desde puntos de vista inusuales que les hace tener una perspectiva particular para formular acciones poco convencionales, ven los obstáculos no como dificultades infranqueables, sino como una oportunidad para generar formas de transponerlos, comportamientos que patentizan la utilización de habilidades de tipo creativa.

\subsection{Conceptos emergentes de 3er orden}

Las habilidades que conforman lo que se ha denominado inteligencia exitosa evidencian el uso del pensamiento que permite la utilización adecuada del conocimiento. El pensamiento conceptualizado por Bourne (1985), como un proceso complejo de múltiples facetas, esencialmente interno que implica representaciones simbólicas y que tiene influencia sobre la conducta manifiesta, hace énfasis en que las representaciones simbólicas que se producen al interior del individuo están asociadas a sus habilidades y que éstas a su vez han sido producto de los procesos de aprendizaje y experiencia propios del pensador. Lo expresa Beauport (1995), las distintas habilidades están conectadas a distintos tipos de pensamiento que son los responsables de la manera en que se recibe, selecciona y evalúa mediante un complejo proceso cognitivo, la información obtenida del mundo exterior la cual está direccionada por el interés del pensador. Este planteamiento sirve de puerta de entrada para incursionar en los tipos de pensamiento que se acoplan de manera concreta a las habilidades demostradas por los empresarios tomados en el estudio. El Cuadro 2 , simboliza la relación existente entre los conceptos emergentes de 1ro, 2do y 3er orden y evidencia la relación entre habilidades y tipos de pensamiento.

El Cuadro 2, muestra las conexiones entre los conceptos emergentes en sus distintos niveles, el último de los cuales está asociado a los tipos de pensamiento que surgen como consecuencia de las habilidades expresadas por los empresarios exitosos. Estos diferentes tipos de pensamiento interactúan entre sí y están direccionados por la visión prospectiva que poseen los empresarios, quienes tienen claramente definido los objetivos y metas que persiguen sus or- 


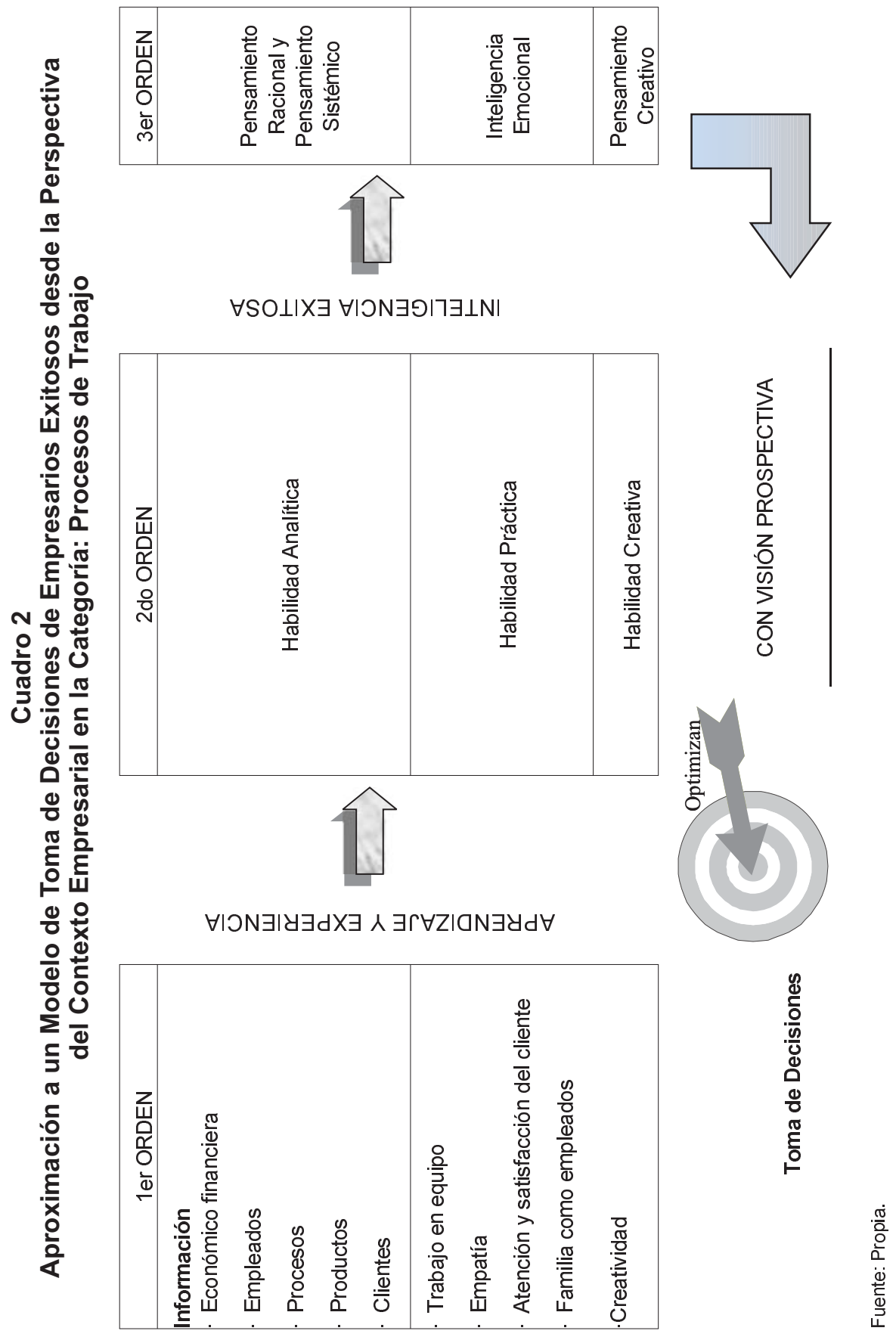


ganizaciones a largo plazo, los cuales a su vez están regidos por la conciencia de la optimización que funge como ente direccionador para su establecimiento y puesta en marcha.

Los elementos representados, están indefectiblemente conectados al proceso decisorio de una manera no solo sistemática, sino sistémica y donde el principio de recursión del pensamiento complejo se manifiesta en toda su magnitud al explicar las relaciones e interacciones que se generan entre estos elementos. EI pensamiento, es de carácter interno y no puede ser observado de manera directa; sin embargo, el mismo pudo ser evidenciado en el análisis de los comentarios, relatos y narraciones de los empresarios exitosos, que describen la manera en que los mismos enfocan las situaciones y problemas, como los analizan, enfrentan y generan alternativas de solución que culminan en decisiones exitosas.

El pensamiento racional, según Beauport (1995:5), "es el proceso por el cual percibimos información por medio de conexiones secuenciales destacando las razones lógicas, la causa y el efecto". Lo secuencial es quizás la característica más obvia de pensamiento racional que implica un análisis paso a paso, una sucesión de operaciones que se preceden unas a otras en un intento de cerrar un proceso antes de incursionar en otro, la realidad es vista de manera lineal lo cual justifica que a este tipo de pensamiento también se le dé ésta denominación. La causa y el efecto están íntegramente conectados en el pensamiento racional, y según la autora citada, son los gemelos de la lógica, la cual requiere de ambos conceptos para poder operar, de hecho, la lógica no es más que la explicación de los efectos a partir de las causas. Esta argumentación justifica de manera evidente e innegable el uso del pensamiento racional por parte de los empresarios exitosos tomados como informantes, dado el uso de la habilidad analítica para el desciframiento de problemas que concluyen en toma de decisiones efectivas, expresadas en acciones concretas observables en su conducta, en una clara expresión física de la habilidad práctica.

Otro tipo de pensamiento ligado a las habilidades analítica y práctica, es el pensamiento sistémico, definido anteriormente como el proceso del entendimiento y la razón que genera respuestas simbólicas para la solución de problemas tomando en consideración los distintos elementos que lo constituyen y las interrelaciones que entre los mismos se generan, dando como resultado decisiones adecuadas al todo dentro de un contexto particular. El pensamiento sistémico se basa, según Ackoff (2000) en la percepción del mundo real en término de totalidades para su análisis, comprensión y accionar, es a todas luces integrador de los distintos elementos de determinada estructura y, las interrelaciones que se generan entre los mismos, a fin de proponer soluciones que sean acordes al sistema como totalidad.

A nivel de decisiones y comportamientos, Beauport (1995), Ferrés (1996) y Goleman (1997), coinciden en afirmar que los mismos son consecuencia del pensamiento lógico: asociado a la racionalidad que funciona por medio de la argumentación y, la inteligencia emocional: asociada al manejo de la emotividad que funciona por asociación. Las emociones 
tienen su asiento en lo que MacLean (citado por Beauport, 1995), denomina cerebro límbico, que representa el cerebro del sentir o de las emociones humanas, el cual interactúa con el cerebro neocortical, en el cual tiene asiento la racionalidad, para generar el desempeño diario de las personas.

La inteligencia emocional, considerada como un tipo especial de pensamiento, está conformada por habilidades que permiten a las personas automotivarse y persistir frente a las decepciones, controlar impulsos y regular el humor, además de proveerlas de una capacidad especial para detectar emociones ajenas y conectarse con ellas. Según Goleman (1999), la inteligencia emocional, determina dos tipos de aptitudes de orden general: aptitudes personales, que determinan el dominio emocional de la misma persona; y aptitudes sociales, determinan el manejo de las relaciones. Las aptitudes personales se subdividen en el autoconocimiento, implica conocer los propios estados internos, preferencias, recursos e intuiciones y está conformado por la conciencia emocional, la autoevaluación precisa y confianza en sí mismo; la autorregulación, que involucra manejo de estados internos, impulsos y recursos y que se estructura con autocontrol, la confiabilidad, la escrupulosidad, la adaptabilidad y la innovación; la motivación, que es una tendencia que guía o facilita la obtención de las metas, incluye el afán de triunfo, el compromiso, la iniciativa y el optimismo, estas aptitudes al ser propias del individuo en su fuero interno le proporcionan una cualidades especiales para asumir acciones concretas para la resolución de problemas y la toma de decisiones.
Las aptitudes de tipo social, establecen el manejo de relaciones interpersonales, está caracterizada por la empatía y las habilidades sociales. La empatía, es una capacidad especial que permite captación de sentimientos, necesidades e intereses ajenos a través de la interpretación de canales no verbales como tono de la voz, ademanes, expresión facial. Steiner (1998:61) la define como "el sexto sentido que permite percibir la energía emocional de la misma manera que el ojo percibe la luz".

Las habilidades sociales representadas por la capacidad para inducir en otros las respuestas que se desean, entre estas habilidades se encuentran: la influencia, que incluye tácticas de persuasión; la comunicación, que implica ser capaz de escuchar, y a la vez emitir mensajes claros y convincentes; el liderazgo, como fuente de inspiración para otros; el manejo de conflictos, conectada a la capacidad de negociar y resolver desacuerdos; la colaboración y cooperación, que implica trabajar con otros para alcanzar metas compartidas la cual está íntimamente relacionada con la habilidad para el trabajo en equipo, que es el responsable de crear la sinergia necesaria para mantener la dirección en pos de las metas.

El análisis de los relatos de los empresarios exitosos, ubicados dentro del contexto empresarial en la categoría procesos de trabajo, denotan una capacidad especial para entrar en sintonía con otros y estrechar relaciones basadas en el conocimiento de sus sentimientos, deseos y aspiraciones, en clara evidencia del manejo de las aptitudes sociales por parte de los entrevistados. La inteligencia emocional es evidente en el comportamiento de 
estos individuos y está relacionada con las habilidades de tipo práctico al ser las emociones los motores que mueven a la acción, tal como lo expresa Torrabadella (1997:21) quien asegura "cuando alguien piensa que debe tomar una decisión, no lo hará hasta que una emoción lo induzca a ello. Las emociones son las que mueven a la acción".

Otro tipo de pensamiento que toma figura al analizar los relatos, es el relacionado con el ingenio, la imaginación y con lo que ha definido De Bono (1995), como pensamiento lateral, el pensamiento creativo que según este autor está relacionado con la capacidad de encontrar otra forma de percibir y procesar la información que se posee para resolver una situación o un problema, y que conlleva una toma de decisiones que no sigue los cursos naturales sino que se desvían hacia los lados creando nuevas pautas de acción. El desempeño creativo es uno de los factores que más contribuye a la solución de problemas, especialmente cuando intervienen aspectos cognoscitivos y se considera la estructura del problema como un modelo de búsqueda para el logro de objetivos concretos, por lo que se exige el uso del análisis del ambiente y la exploración de la memoria.

El pensamiento creativo, confiere una habilidad especial para percibir y procesar la información que provee el medio, que conlleva a afrontar las situaciones y problemas de una manera particular y si se quiere personal, para encontrar vías de acción únicas que propendan al logro de objetivos previamente determinados. La habilidad creativa propia de este tipo de pensamiento está expresada en las acciones registradas por los relatos de los empresarios exitosos, y hacen patente el uso del pensamiento creativo en la manera en como enfrentan o afrontan las distintas situaciones a las que se ven sometidos en su labor de gerentes de negocio.

\section{Conclusión}

Los empresarios exitosos tomados como informantes, dan cuenta de una serie de capacidades especiales para el aprendizaje y manejo de la experiencia que se manifiestan en habilidades analíticas, prácticas y creativas propias de un tipo especial de inteligencia, como lo es la inteligencia exitosa, la cual les dá unas características particulares al empresario para percibir oportunidades de negocio, analizarlas cuantitativa y cualitativamente, ejecutar las acciones propias para la implementación y desarrollo de la oportunidad, agregando valor a la misma con propuestas no convecionales que aseguran la consolidación del negocio.

Las habilidades confluyen en distintos tipos de pensamiento que demuestran la conexión entre los procesos cognitivos y la acción y que, en este caso particular, se evidencian en el pensamiento racional, sistémico, creativo y la inteligencia emocional (tomada como un tipo especial de pensamiento). La relación sistemática y sistémica de las habilidades y tipos de pensamiento, generan un tomador de decisiones efectivo con visión prospectiva, que busca en todo momento la optimización de los recursos que posee en un momento y contexto particular. Esto demuestra que no solo la racionalidad es la responsable de la toma de decisiones efectiva, sino la conjugación del 
pensamiento sistémico que propicia la interconexión de percepciones, informaciones, eventos de distinta índole que amplian el marco de referencia para una decisión efectiva. Adicionalmente, el ingrediente de conexión personal e interpersonal que garantiza el autocontrol, la automotivación y las relaciones interpersonales efectivas que generan una capacidad especial para la negociación, ingrediente especial que augura toma de decisiones grupales efectivas.

En definitiva, los empresarios exitosos tomados como informantes del estudio, asumen la toma de decisiones bajo la influencia de los diferentes tipos de pensamiento, que han sido desarrollados a partir de las habilidades manifiestas en sus acciones, las cuales han sido reguladas por los procesos de aprendisaje y experiencia producto de la práctica empresarial.

Es necesario acotar que aunque la explicación de este planteamiento teórico es lineal, en realidad cada uno de los componentes del proceso incide sobre los otros en un continuo ir y venir, convirtiéndose a la vez en productores y generadores de sí mismos y de otros componentes.

\section{Referencias bibliográficas}

Albornoz, José Hernán (1997). Diccionario de Filosofía. Venezuela: Editores Vadell Hermanos.

Anderson, John (2001). Aprendizajey Memoria. Un Enfoque Integral. México: McGraw Hill.

Ackoff, Russell (2000). El Arte de Resolver Problemas. [Informe en Línea] Disponible: ¡Error! Referencia de hiper- vínculo no válida.[Consulta:2003, noviembre 05].

Bandura, Alberth (1983). Aprendizaje Social y Desarrollo de la Personalidad. Madrid: Alianza Editorial.

Beauport, Elaine (1995). Las Tres Caras de la Mente. Venezuela: Galac Editores.

Benjafield, John (1997). Cognition. Estados Unidos de América: Prentice Hall.

Berlyne, Daniel (1982). Estructura y Función del Pensamiento. México: Editorial Trillas.

Bourne, Lyle (1985). Psicología del Pensamiento. México: Editorial Trillas.

Castillo, Elizabeth (2002). Toma de Decisiones. Un enfoque Particular. Trabajo de ascenso no publicado. UNET. San Cristóbal.Venezuela

Contreras, Ángel (2001). Presentación de Modelos Teórico Conceptuales. La Teoría del Aprendizaje Social. Disponible: http://www.comminit.com/la/ lapm/sid-404.html. [Consulta: 2004, enero 08].

Covey, Stephen (1993). Los 7 Hábitos de la Gente Altamente Efectiva. México: Editorial Paidós Mexicana.

Daft, Richard (2000). Teoría y Diseño Organizacional. México: Thomson Editores.

De Bono, Edward (1995). El Pensamiento Creativo. EI Poder del Pensamiento Lateral para la Creación de Nuevas Ideas. España: Ediciones Paidós.

Escorche, Victor, Guzmán, José., Medina, Ernesto (1995). Manual del Consultor en Productividad y Calidad. Venezuela: Fim-Productividad-Corporación Andina de Fomento.

Ferrés, Joan (1996). Las Dos Grandes Vías de la Comunicación Persuasiva. España: Ediciones Paidós.

Gabiña, Juanjo (1996). El Futuro Revisitado. La reflexión Prospectiva como Arma de Estrategia y de Decisión. Colombia: Alfaomega Ediciones. 
Goleman, Daniel (1997). La Inteligencia Emocional. Argentina: Javier Vergara Editor S.A.

Goleman, Daniel (1999). La Inteligencia Emocional en la Empresa. Argentina: Javier Vergara Editor S.A.

Godet, Michell (1995). De la Anticipación a la Acción. Manual de Prospectiva y Estrategia. Colombia: Alfaomega Ediciones

Gosling, Louisa (1995). Toolkits. A Practical Guide to Assessment, Monitoring, Review and Evaluation. Washington: SDC.

Hernández, Sergio (2002). Administración. Pensamiento, Proceso, Estrategia y Vanguardia. México: McGraw Hill.

Harrington, James (1990). Como Incrementar la Calidad y la Productividad. México: McGraw Hill.

Instituto Andino de Sistemas (2002). ¿Qué es Pensamiento Sistémico? Disponible: http://www.iasvirtual.net. [Consulta: 2004, febrero 23].

Horngren, Gary (1989).Contabilidad Financiera. Introducción. México: Prentice Hall.

Kotler, Phillips (1992). Dirección de Mercadotecnia, Análisis, Planeación, Implementación y Control. ( $\left.6^{\mathrm{a}} \mathrm{Ed}\right)$. México: McGraw Hill.

Martínez, Juan (2001). ¿Qué es Economía? (2 $\left.{ }^{\mathrm{a}} \mathrm{Ed}\right)$. España. Editorial Emvi.

Märtin, Doris (1998). EQ Que es Inteligencia Emocional. Como Lograr que las Emociones Determinen Nuestro Triunfo en Todos los Ámbitos de la Vida. Madrid: Edaf.

Mojica. Francisco (1991). La Prospectiva. Técnica para Visualizar el Futuro. Colombia: Fondo Editorial Legis.

Niebel, Benjamin (1996). Ingeniería Industrial: Estudio de Tiempos y Movimientos. México: Alfaomega Editores.
Observatorio Pyme (2001). Estudio de la Pequeña y Mediana Empresa en Venezuela Programa Bolívar. Caracas: Corporación Andina de Fomento.

Radler, J. (2001). Cognitive Perspective of Human Intelligence. Disponible: http//www.fau.edu/divdept/cla/honor/ HTML/Projectl.html [Consulta: 2004, marzo 19].

Robbins, Stephen (1999). Comportamiento Organizacional: Conceptos, Controversias y Aplicaciones. ( $\left.8^{\mathrm{a}} \mathrm{Ed}\right)$. México: Prentice Hall.

Salgado, Mónica (2000). Introducción al SGQ ISO 9000 Versión 2000. España: Ediciones Barés.

Sánchez, Margarita (1994). Aprende a Pensar. Organización del Pensamiento. México: Editorial Trillas.

Sánchez, Margarita (1998). Desarrollo de Habilidades del Pensamiento. Razonamiento Verbal y Solución de Problemas. México: Editorial Trillas.

Sánchez, Margarita (1999). Desarrollo de Habilidades del Pensamiento. Creatividad. México: Editorial Trillas.

Sandin, María (2003). Investigación Cualitativa en Educación. Fundamentos y Tradiciones. Madrid: Mc. Graw Hill.

Stanton, William (1991). Fundamentos de Marketing. México: McGraw Hill.

Sternberg, Robert (1994). Intelligence and its Measurement. Journal of Personality and Social Psychology. E.U.

Sternberg, Robert (1997). Inteligencia Exitosa. Como una Inteligencia Práctica y Creativa Determinan el Éxito en la Vida. España: Paidós.

Sternberg, Robert (1998). Beyonnd I.Q. The Triarquial Theory of Human Inteligence. Hillsdale, N.J. Erlbuam.

Steiner, Claude (1998). La Educación Emocional. Argentina: Vergara Editor. 
Stoner, James; Freeman, Edward. y Gilbert, Daniel (1996). Administración. (6 ${ }^{a}$ Ed). México: Prentice Hall.

Stoner, James. Freeman, Edward. y Gilbert, Daniel (1998). Administración. (7 ${ }^{\mathrm{a}}$ Ed). México: Prentice Hall.
Torrabadella, Paul (1997). Como Desarrollar la Inteligencia Emocional. Barcelona: Producciones Grales. 